\title{
Rotation of two trapped microparticles in vacuum: observation of optically mediated parametric resonances
}

\author{
Yoshihiko Arita ${ }^{1}$, Michael Mazilu ${ }^{1}$, Tom Vettenburg ${ }^{1}$, Ewan M. Wright ${ }^{2}$, and Kishan \\ DHOLAKIA ${ }^{1,2^{*}}$ \\ ${ }^{1}$ SUPA, School of Physics and Astronomy, University of St Andrews, St Andrews, Fife, KY16 9SS, United Kingdom \\ ${ }^{2}$ College of Optical Sciences, The University of Arizona, 1630 East University Boulevard, Tucson, AZ 85721-0094, USA \\ *Corresponding author: kd1@st-andrews.ac.uk
}

Compiled September 15, 2015

We demonstrate trapping and rotation of two mesoscopic particles in vacuum using a spatial light modulator based approach to trap more than one particle, induce controlled rotation of individual particles and mediate inter-particle separation. By trapping and rotating two vaterite particles, we observe intensity modulation of the scattered light at the sum and difference frequencies with respect to the individual rotation rates. This first demonstration of optical interference between two microparticles in vacuum leads to a platform to potentially explore optical binding and quantum friction effects. (- 2015 Optical Society of America

OCIS codes: (140.7010) Laser trapping; (350.4855) Optical tweezers or optical manipulation; (190.2620) Harmonic generation and mixing.

http://dx.doi.org/10.1364/ol.XX.XXXXXX

An emerging theme in quantum optomechanics is the trapping and cooling of mesoscopic particles in vacuum enabled by the absence of direct dissipation and any physical contact. This approach uses the light-matter interaction and linear momentum transfer from the incident light field to the trapped object to cool its centre-of-mass with the goal of attaining the quantum ground state [1-5]. Though yet to be attained, recent advances have shown a number of cooling mechanisms at play and millikelvin temperatures have been reached [6]. Furthermore, fascinating insights into Brownian dynamics have been seen [7]. Our previous work in this area showed the transfer of spin angular momentum to a trapped birefringent particle recording rotation rates of up to $10 \mathrm{MHz}$ [8].

For a number of key studies, such as multi-particle entanglement and vacuum friction $[9,10]$, the ability to individually trap and move multiple particles in vacuum with complete freedom over the inter-particle spacing would be crucial. These goals can be attained by the ability to shape and control the optical trapping field in phase, amplitude and polarisation. To realise these major goals would add additional degrees of freedom and open up new studies in this burgeoning area. Indeed, control of trapping more than one particle in a vacuum environment critically opens up new prospects. This includes particle-particle interactions such as optical binding [11]. Such binding can lead to new co-operative effects between particles and potentially a route for cooling translational degrees of freedom for multiple objects simultaneously. In terms of studies of vacuum rotational friction and associated effects, controlled positioning of two or more objects can lead to powerful new embodiments of proposed experiments [10]. We note that standing wave traps can hold more than one particle for vacuum studies [12].

In this letter, we demonstrate the first optical linear and spin momentum transfer to two micro-particles independently trapped at close distance in vacuum. Aided by a dynamic diffractive optical element, we are able to create traps with precise spatial separation and set particles into counter- or co-rotation by tuning the individual spin angular momentum transfer for each particle. The system may be readily extended to trap more than two microparticles. We observe inter-particle interactions, in the form of sum and difference frequency mixing, encoded onto the scattered light. Our observations are confirmed by a numerical model.

We have phenomenologically modelled the power spectrum arising from the inter-particle scattering of the two birefringent particles. The model considers multiple scattering of the incident beam between the two rotating particles described by the scattering coefficients $S_{12}$ and $S_{21}$. We assume these coefficients to depend on the instantaneous orientation of the particle

$$
S_{12}(\theta)=a_{0}+a_{1} \sin \left(\theta+\phi_{1}\right)+a_{2} \sin \left(2 \theta+\phi_{2}\right)+\ldots
$$

where $\theta$ represents, for instance, the angle of the optical axis around the rotation direction. In a similar way one can define the scattering term $S_{21}$. The Fourier coefficients $a_{i}$ for $i>0$ correspond to the optical asymmetry of the particle due to its departure from spherical shape, optical inhomogeneity and birefringent anisotropy. For example, the first term $\left(a_{1}\right)$, which delivers the modulation of the scattering field at the fundamental rotation frequency, could be related to a small single sided optical asymmetry which perturbs the scattered field once every full revolution. Further, the second term $\left(a_{2}\right)$ is related to the 
particle birefringence and modulates the scattered field at twice the rotation frequency. In this approach, we need to consider four scattering events, each with different Fourier coefficients, namely: $S_{1 \infty}(\theta), S_{2 \infty}(\theta), S_{12}(\theta)$ and $S_{21}(\theta)$ corresponding respectively to the scattering from the first particle towards the far-field and towards the second particle and from the second particle towards the far-field and towards the first particle. The total scattering measured is then described by

$$
\begin{aligned}
S= & S_{1 \infty}\left(\theta_{1}\right)+S_{12}\left(\theta_{1}\right) S_{2 \infty}\left(\theta_{2}\right) \\
& +S_{2 \infty}\left(\theta_{2}\right)+S_{21}\left(\theta_{2}\right) S_{1 \infty}\left(\theta_{1}\right)+\ldots
\end{aligned}
$$

Higher order multiple scattering terms can be taken into account; however, their individual contributions decrease geometrically with order.

To calculate the power spectrum of the scattered intensity (Fig.1), we consider two birefringent particles that have different diameters ( $1 \%$ variation) which induces different optical torques. Their levitated trap, the torques and the overall gyroscopic evolution of the particles are described in the single particle experiment [8]. In brief, as the particles rotate, their form asymmetries and optical anisotropies modulate, at the rotation frequency and its harmonics, the incident light field akin to a macroscopic Raman effect. Here, we considered asymmetry upto the $a_{2}$ term in equation (1). Multiple scattering between the two particles further delivers a heterodyne signal. This signal displays the variety of parametric resonances that are observed in the experiment, see Fig.1.

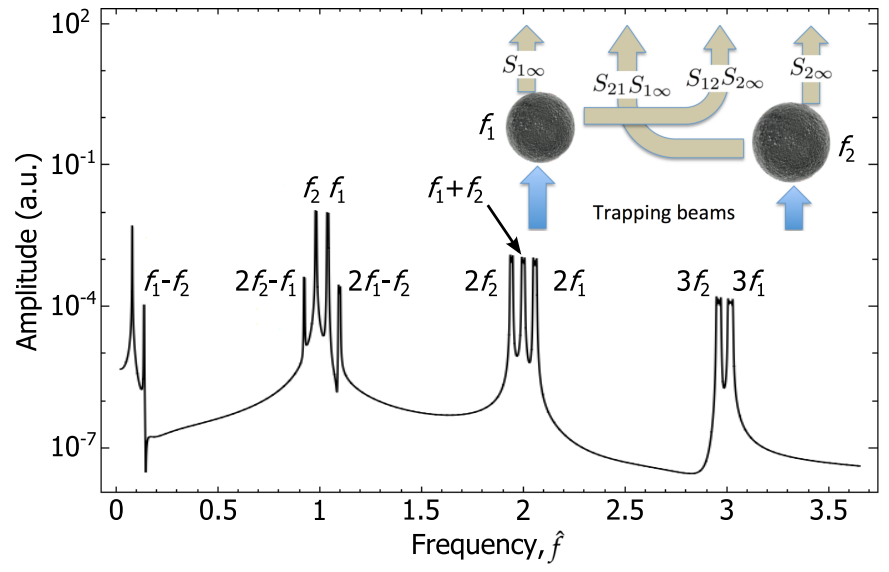

Fig. 1. Numerical simulation of the power spectra of the scattered light from two particles trapped in vacuum rotating at slightly different frequencies of $f_{1}$ and $f_{2}$, where $\hat{f}=\left(f_{1}+f_{2}\right) / 2$. Inset shows the diagram of the scattering process. The data set can be accessed at [13].

Experimentally, the rotation of microparticles is achieved by trapping birefringent particles with either a left or right circularly polarized focus beam. Vaterite crystals are positive uniaxial birefringent material with a spherical morphology. We synthesise a mono-disperse sample of vaterite with a mean diameter of $4.4 \mu \mathrm{m}$ with two standard deviations $(2 \sigma)$ of $\pm 0.1 \mu \mathrm{m}$ [18].

Fig. 2 shows the schematic of the vacuum trapping system, which employs a holographic optical tweezers based on the setup used for the previous trapping and rotation experiments in air [14] and in vacuum [8]. To allow for individual intensity control and three-dimensional positioning of two traps, the active surface of a spatial light modulator (SLM, Hamamatsu LCOS $\mathrm{X} 10468-03)$ is conjugate to the back aperture of the objective.
Independent polarisation control of traps is achieved by generating beams of orthogonal polarisation states which are subsequently combined at a polarising beam displacer (PBD, Thorlabs, BD27) [15]. This is a variant of a previous approach [16] that requires two optical paths together with a "split-screen" SLM. In our approach two first-order diffraction beams are generated by the SLM with their beam separation matched with the designed separation of the PBD where the two beams are recombined. The common-path design of the interferometer in this way reduces its sensitivity to environmental effects, and phase shifting and enables precise control over the polarisation state of each trap. Any aberration, including that caused by the divergence of the transmitted beam is compensated for by the SLM with the help of an auxiliary camera at a plane conjugate with the sample [17]. The axial positions of each trap can be individually controlled by adding a parabolic phase change to the grating, which is effectively a Fresnel lens. The deflection efficiency can be adjusted for each first-order beam to control the optical power of each trap.

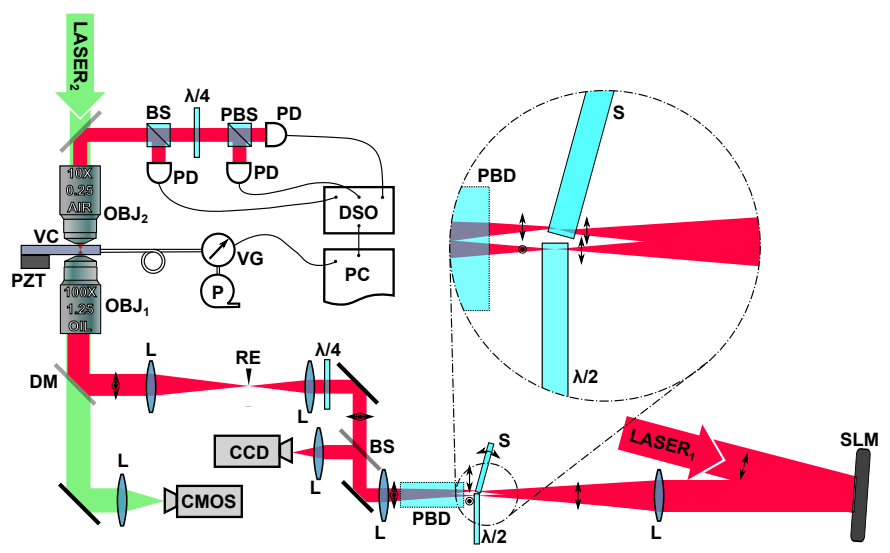

Fig. 2. Schematic of the experimental set-up. Labels denote the continuous wave $1070 \mathrm{~nm}$ trapping laser $\left(\mathrm{LASER}_{1}\right)$, half-wave plate $(\lambda / 2)$, compensator $(S)$, dichroic mirror (DM), quarterwave plate $(\lambda / 4)$, beam splitter (BS), photodiodes (PD), nanosecond laser $\left(\mathrm{LASER}_{2}\right)$, fast imaging device (CMOS), digital storage oscilloscope (DSO), vacuum chamber (VC), piezo electric transducer (PZT), vacuum gauge (VG), vacuum pump (P).

As circular polarisation purity is of utmost importance in our experiment, a quarter wave plate is introduced between the PBD and the microscope objective (Nikon, E Plan $100 \times$, NA $=1.25$ ), converting linear polarisation to circular, whilst linear polarisation is achieved by coherently combining a left and a right polarised focus with identical amplitude at the same position in the sample chamber. The sample plane is imaged in reflection through the objective using a fast CMOS camera (Mikrotron, MC-1362) synchronised with single nanosecond laser pulse illumination (Elforlight, SPOT, $532 \mathrm{~nm}$ ). The forward scattered beam after transmission through the rotating vaterite particles is collected by a condenser lens, which is directed to a series of beam splitter cubes one for unpolarised component and the other for polarised left-/right- circularly polarised components of the beam. Three fast photodiodes (Thorlabs, DET10C) are used to measure the light intensity of each component of the beam modulated by those two rotating particles [8].

We designed a miniature vacuum chamber, which has two optical round glass windows (Harvard Apparatus, CS-8R, thick- 
ness of $150 \mu \mathrm{m}$ ) compatible with the high numerical aperture objective used. Microparticles of interest are dried in advance and preloaded onto the bottom glass window of the chamber, which is affixed to a piezoelectric transducer (APC International, Cat. no.70-2221) to launch microparticles into the traps.

In the experiment, two particles were initially trapped in air (at a pressure of $10^{5} \mathrm{~Pa}$ ) by a continuous wave $1070 \mathrm{~nm}$ laser with a total power of $25 \mathrm{~mW}$ (measured at the back aperture of the objective, transmission $58 \%$ ), which is equally shared between each trap site. The rotation rate of each particle may be different as it is dependent on the particle size, which in turn affects the optical torques as well as the Stokes drag torques. The distance between the equators of the two particles can be as small as $2 \mu \mathrm{m}$ without losing either of the particles from their trap sites at atmospheric pressure. The pressure of the chamber is gradually reduced to $10^{-1} \mathrm{~Pa}$ while recording the forward scattered light intensity using fast photodiodes. The intensity of this forward scattered light is modulated by any asymmetries of the rotating spheres and we observe modulation frequencies, $f_{1}$ and $f_{2}$ corresponding to the rotation frequencies of the first and second particles. As previously observed, we also detect a modulation at twice the rotation frequencies, $2 f_{1}$ and $2 f_{2}$ [8].

For a single trapped, rotating particle, it is straightforward to identify its mechanical modes relating to transverse oscillation and rotation. Fig. 3a shows the position power spectrum of a single particle trapped and rotating at a pressure of $16.0 \mathrm{~Pa}$. The lateral and axial modes of oscillation frequencies are found to be $f_{\mathrm{xy}}=663 \mathrm{~Hz}$ and $f_{\mathrm{z}}=421 \mathrm{~Hz}$ together with their higher harmonics, e.g. $2 f_{\mathrm{xy}}, 3 f_{\mathrm{xy}}$ and $2 f_{\mathrm{z}}$, respectively. The particle rotates at a frequency of $f_{1}=13 \mathrm{kHz}$, where sidebands separated by the translational mode frequency of $f_{\mathrm{xy}}=663 \mathrm{~Hz}$ are also present (Fig. 3a inset). This is because the translational motion modulates the rotational motion of a particle such that these mechanical modes are parametrically coupled [8]. High harmonics of the rotational frequency, $f_{1}$ are observable up to five overtones in the power spectrum.

When a second particle is present and rotating at a close surface to surface distance of $6 \mu \mathrm{m}$ measured between the equators (e.g. Visualisation 1, recorded at a pressure of $985.3 \mathrm{~Pa}$, which is rendered to 25 frames per second from the original rate of 3,493 frames per second) we observe a complex power spectrum (Fig. 3b,c) showing the generation of multiple modulation frequencies. One notable feature of the dynamics of the two rotating particles is the heterodyne frequency generation involving the individual rotation frequencies of each particle, $f_{1}$ and $f_{2}$. This does not mean just addition of two power spectra but notably includes mixing of the rotational frequencies of each particle yielding sum and difference frequency generation (DFG). Table 1 identifies such parametric resonances corresponding to the peaks found in Fig. 3b,c. For example, the peak at $6,637 \mathrm{~Hz}$ corresponds to $2\left(f_{1}-f_{2}\right)$ and is only observable when a second rotating particle is present. In a similar manner, we obtained the power spectrum from two counter-rotating particles, in which case both sum and DFG were also observed. Here it should be noted that if the two beams possess the same polarisation, i.e. two particles co-rotate, trapping beam interference may occur and alters the beam power at each trap depending on the separation of the two beams.

We further investigate a series of power spectra obtained from two co-rotating particles at different pressures ranging from $10^{5} \mathrm{~Pa}$ (atmospheric pressure) to $10^{-1} \mathrm{~Pa}$. Two vaterite particles are trapped and rotated at rates of $f_{1}$ and $f_{2}$ with a surface to surface distance of $3 \mu \mathrm{m}$ (see Fig.4a inset and Visu-

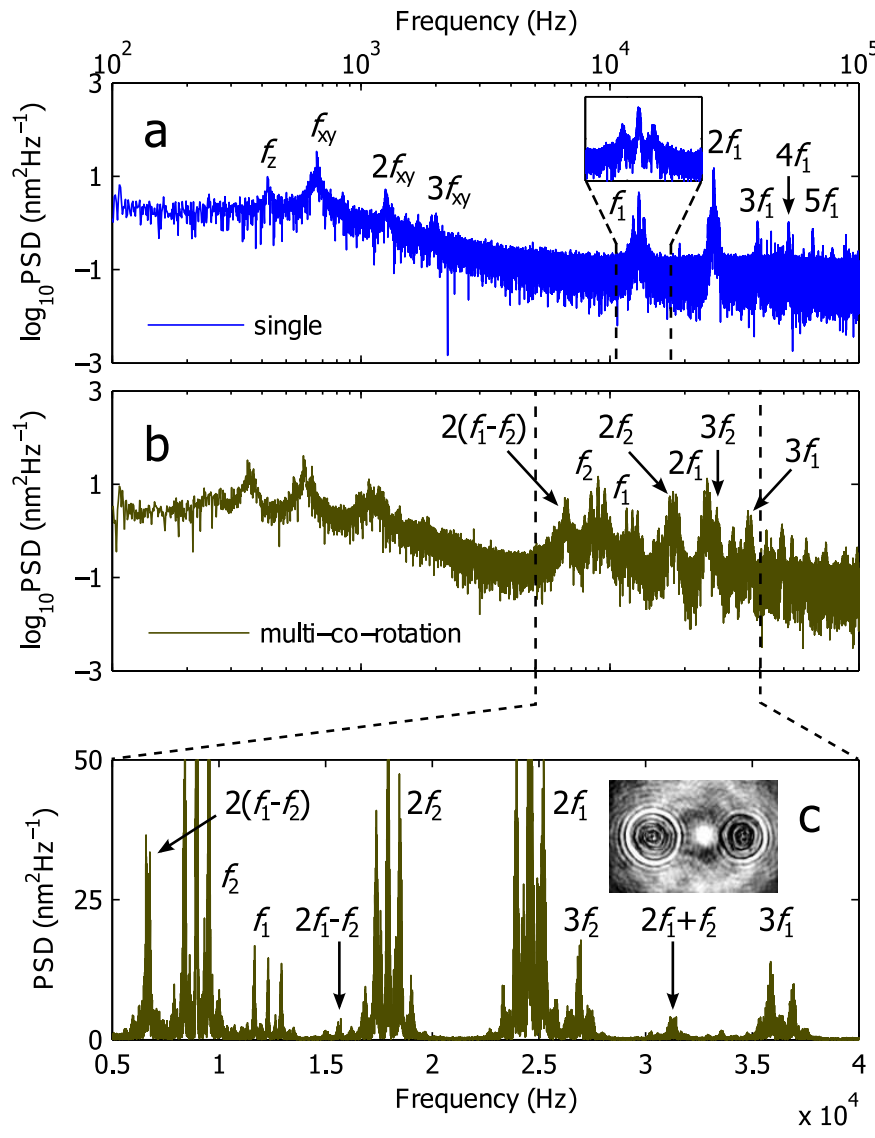

Fig. 3. Power spectra of a single-/multi-particle rotating at a pressure of $16 \mathrm{~Pa}$. (a) A single particle rotating. (b) Two particles co-rotating with the distance of $6 \mu \mathrm{m}$ between the equators. (c) An expanded view of the selected region of (b) linearly scaled. Inset shows a snapshot of the two particle rotating, where the field of view is $21 \mu \mathrm{m} \times 14 \mu \mathrm{m}$. The data set can be accessed at [13].

Table 1. Modulation frequencies corresponding to Fig.3b,c. $f_{1}$ and $f_{2}$ denote the rotation frequencies of particle 1 and of particle 2 .

\begin{tabular}{cccccc}
\hline$f(\mathrm{~Hz})$ & Mode & $f(\mathrm{~Hz})$ & Mode & $f(\mathrm{~Hz})$ & Mode \\
\hline 6,637 & $2\left(f_{1}-f_{2}\right)$ & 17,950 & $2 f_{2}$ & 35,860 & $4 f_{2}$ \\
8,960 & $f_{2}$ & 24,570 & $2 f_{1}$ & 36,930 & $3 f_{1}$ \\
12,320 & $f_{1}$ & 26,980 & $3 f_{2}$ & 42,490 & $2\left(f_{1}+f_{2}\right)$ \\
15,710 & $2 f_{1}-f_{2}$ & 33,550 & $2 f_{1}+f_{2}$ & 44,860 & $5 f_{2}$ \\
\hline
\end{tabular}

alisation 2, recorded at a pressure of $414.6 \mathrm{~Pa}$ and rendered as Visualisation 1). Rotational mode frequencies of the two particles, namely $f_{1}, f_{2}, 2 f_{1}, 2 f_{2}$ and the DFG signal of $2\left(f_{1}-f_{2}\right)$ are tracked for each power spectrum and plotted in Fig.4a as a function of the damping coefficient, $\Gamma(P)=8 \pi \mu(P) r^{3}$, where $\mu(P)$ denotes the viscosity and is measured using a single particle, subject to a pressure independent optical torque, thus rotating at a terminal frequency $f(P)$. At this rotation frequency we have $\mu(P) \cdot f(P)=f\left(10^{5} \mathrm{~Pa}\right) \cdot \mu\left(10^{5} \mathrm{~Pa}\right)$ with $\mu\left(10^{5} \mathrm{~Pa}\right)=18.2 \times 10^{-6}$ Pas. 

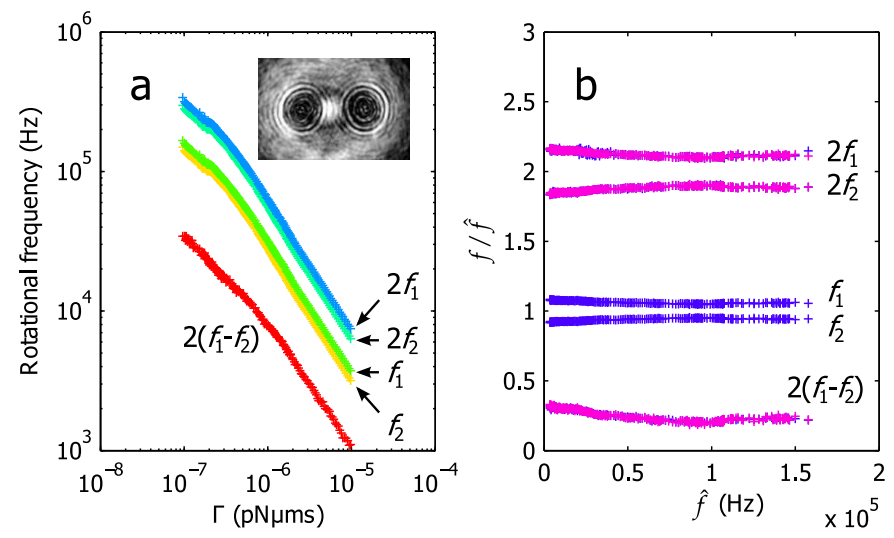

Fig. 4. Rotational frequencies of the two co-rotating particles at different pressures. (a) Rotational frequencies for different damping coefficients. Inset shows a snapshot of the two rotating particles, where the field of view is $21 \mu \mathrm{m} \times 14 \mu \mathrm{m}$. (b) Rotational frequencies normalized by the mean rotation rate, $\hat{f}=\left(f_{1}+f_{2}\right) / 2$ and plotted as a function of $\hat{f}$ (blue). The deduced frequencies calculated from the fundamental rotational frequencies of $f_{1}$ and $f_{2}$ are plotted in red. The data set can be accessed at [13].

To understand if there are any inter-particle dynamics present, these modulation frequencies including the DFG signal are normalised by the mean rotation rate, $\hat{f}=\left(f_{1}+f_{2}\right) / 2$, and plotted in blue (Fig. $4 \mathrm{~b}$ ) as a function of the mean rotation rate, $\hat{f}$. Figure $4 \mathrm{~b}$ as such presents the data in a way that eliminates the dependence on $\Gamma$. In Figure $4 \mathrm{~b}$ we observe that the nominal second harmonic frequency (in blue) is equal to the double of the measured fundamental frequency (in red) for each particle. In the same manner, we plot the DFG signal, $2\left(f_{1}-f_{2}\right)$ which is determined by the relative rotation rates of the two particles. It is this DFG signal presented in figure $4 \mathrm{~b}$ that may be used to detect inter-particle interactions such as binding. If this signal were to only depend on $\Gamma$, the normalised DFG signal should be constant over any $\hat{f}$. Interestingly, the deviation from a constant value suggests that the motion of these two particles is not independent from each other. A detailed investigation of this will be presented in future.

In summary, we have demonstrated the transfer of both linear and spin angular momentum to two microparticles at close distance in vacuum. The individual control of angular momentum transfer to each traps as well as control over the spatial separation of the each trap was demonstrated. This can serve as a fundamental physics test-bed potentially addressing open questions related to mesoscopic quantum entanglement and vacuum friction, provided inter-particle cooling [3] or sufficiently high rotation rates can be achieved $[9,10]$. We have measured macroscopic Raman-like modulation of the incident light field originating from the rotation of each particle and the subsequent generation of sum- and difference-frequencies.

Optical binding refers to an optically mediated inter-particle interaction that creates new equilibrium positions for closely spaced particles [19-23]. Our system opens up the possibility of observing such optical binding and coupling associated with the observed multiple scattering of light between levitated microparticles in vacuum.

\section{FUNDING INFORMATION}

We thank the UK Engineering and Physical Sciences for funding through grant EP/J01771X/1.

\section{REFERENCES}

1. I. Wilson-Rae, N. Nooshi, W. Zwerger, and T. J. Kippenberg, Phys. Rev. Lett. 99, 093901 (2007).

2. S. Groblacher, J. B. Hertzberg, M. R. Vanner, G. D. Cole, S. Gigan, K. C. Schwab, and M. Aspelmeyer, Nat. Phys. 5, 485-488 (2009).

3. P. F. Barker, Phys. Rev. Lett. 105, 073002 (2010).

4. A. D. O'Connell, M. Hofheinz, M. Ansmann, R. C. Bialczak, M. Lenander, E. Lucero, M. Neeley, D. Sank, H. Wang, M. Weides, J. Wenner, J. M. Martinis, and A. N. Cleland, Nature 464, 697-703 (2010).

5. J. Chan, T. P. M. Alegre, A. H. Safavi-Naeini, J. T. Hill, A. Krause, S. Gröblacher, M. Aspelmeyer, and O. Painter, Nature 478, 89-92 (2011).

6. T. C. Li, S. Kheifets, and M. G. Raizen, Nat. Phys. 7, 527-530 (2011).

7. T. C. Li, S. Kheifets, D. Medellin, and M. G. Raizen, Science $\mathbf{3 2 8 ,}$ 1673-1675 (2010).

8. Y. Arita, M. Mazilu, and K. Dholakia, Nat. Commun. 4, 2374 (2013).

9. A. Manjavacas and F. J. G. de Abajo, Phys. Rev. Lett. 105, 113601 (2010).

10. R. K. Zhao, A. Manjavacas, F. J. G. de Abajo, and J. B. Pendry, Phys. Rev. Lett. 109, 123604 (2012).

11. O. Brzobohaty, T. Cizmar, V. Karasek, M. Siler, K. Dholakia, and P. Zemanek, Opt. Express 18, 25389-25402 (2010).

12. T. S. Monteiro, J. Millen, G. A. T. Pender, F. Marquardt, D. Chang, and P. F. Barker, New J. Phys. 15, 015001 (2013).

13. Y. Arita, M. Mazilu, T. Vettenburg, E. M. Wright, and K. Dholakia, University of St Andrews, http://dx.doi.org/10.17630/ff668806-7a594a3f-866b-ed9d02da10c4 (2015).

14. Y. Arita, A. W. McKinley, M. Mazilu, H. Rubinsztein-Dunlop, and K. Dholakia, Anal. Chem. 83, 8855-8858 (2011).

15. M. Plöschner, T. Tyc, and T. Čižmár, Nat. Photon. 9, 529-535 (2015).

16. D. Preece, S. Keen, E. Botvinick, R. Bowman, M. Padgett, and J. Leach, Opt. Express 16, 15897-15902 (2008).

17. T. Čižmár, M. Mazilu, and K. Dholakia, Nat. Photon. 4, 388-394 (2010).

18. R. Vogel, M. Persson, C. Feng, S. J. Parkin, T. A. Nieminen, B. Wood, N. R. Heckenberg, and H. Rubinsztein-Dunlop, Langmuir 25, 1167211679 (2009).

19. K. Dholakia and P. Zemanek, Rev. Mod. Phys. 82, 1767 (2010).

20. S. A. Tatarkova, A. E. Carruthers, and K. Dholakia, Phys. Rev. Lett. 89, 283901 (2002).

21. M. M. Burns, J. M. Fournier, and J. A. Golovchenko, Science 249, 749-754 (1990).

22. M. Guillon, O. Moine, and B. Stout, Phys. Rev. Lett. 96, 143902 (2006).

23. M. Guillon and B. Stout, Phys. Rev. A 77, 023806 (2008). 


\section{REFERENCES}

1. I. Wilson-Rae, N. Nooshi, W. Zwerger, and T. J. Kippenberg, "Theory of ground state cooling of a mechanical oscillator using dynamical backaction," Phys. Rev. Lett. 99, 093901 (2007).

2. S. Groblacher, J. B. Hertzberg, M. R. Vanner, G. D. Cole, S. Gigan, K. C. Schwab, and M. Aspelmeyer, "Demonstration of an ultracold micro-optomechanical oscillator in a cryogenic cavity," Nat. Phys. 5, 485-488 (2009).

3. P. F. Barker, "Doppler cooling a microsphere," Phys. Rev. Lett. 105, 073002 (2010).

4. A. D. O'Connell, M. Hofheinz, M. Ansmann, R. C. Bialczak, M. Lenander, E. Lucero, M. Neeley, D. Sank, H. Wang, M. Weides, J. Wenner, J. M. Martinis, and A. N. Cleland, "Quantum ground state and single-phonon control of a mechanical resonator," Nature 464, 697703 (2010).

5. J. Chan, T. P. M. Alegre, A. H. Safavi-Naeini, J. T. Hill, A. Krause, S. Gröblacher, M. Aspelmeyer, and O. Painter, "Laser cooling of a nanomechanical oscillator into its quantum ground state," Nature $\mathbf{4 7 8}$, 89-92 (2011).

6. T. C. Li, S. Kheifets, and M. G. Raizen, "Millikelvin cooling of an optically trapped microsphere in vacuum," Nat. Phys. 7, 527-530 (2011).

7. T. C. Li, S. Kheifets, D. Medellin, and M. G. Raizen, "Measurement of the instantaneous velocity of a brownian particle," Science $\mathbf{3 2 8}$, 1673-1675 (2010).

8. Y. Arita, M. Mazilu, and K. Dholakia, "Laser-induced rotation and cooling of a trapped microgyroscope in vacuum," Nat. Commun. 4, 2374 (2013).

9. A. Manjavacas and F. J. G. de Abajo, "Vacuum friction in rotating particles," Phys. Rev. Lett. 105, 113601 (2010).

10. R. K. Zhao, A. Manjavacas, F. J. G. de Abajo, and J. B. Pendry, "Rotational quantum friction," Phys. Rev. Lett. 109, 123604 (2012).

11. O. Brzobohaty, T. Cizmar, V. Karasek, M. Siler, K. Dholakia, and P. Zemanek, "Experimental and theoretical determination of optical binding forces," Opt. Express 18, 25389-25402 (2010).

12. T. S. Monteiro, J. Millen, G. A. T. Pender, F. Marquardt, D. Chang, and P. F. Barker, "Dynamics of levitated nanospheres: towards the strong coupling regime," New J. Phys. 15, 015001 (2013).

13. Y. Arita, M. Mazilu, T. Vettenburg, E. M. Wright, and K. Dholakia, "Data underpinning: Rotation of multiple trapped microparticles in vacuum: observation of parametric optical interactions," University of St Andrews, http://dx.doi.org/10.17630/ff668806-7a59-4a3f-866bed9d02da10c4 (2015).

14. Y. Arita, A. W. McKinley, M. Mazilu, H. Rubinsztein-Dunlop, and K. Dholakia, "Picoliter rheology of gaseous media using a rotating optically trapped birefringent microparticle," Anal. Chem. 83, 8855-8858 (2011).

15. M. Plöschner, T. Tyc, and T. Čižmár, "Seeing through chaos in multimode fibres," Nat. Photon. 9, 529-535 (2015).

16. D. Preece, S. Keen, E. Botvinick, R. Bowman, M. Padgett, and J. Leach, "Independent polarisation control of multiple optical traps," Opt. Express 16, 15897-15902 (2008)

17. T. Čižmár, M. Mazilu, and K. Dholakia, "In situ wavefront correction and its application to micromanipulation," Nat. Photon. 4, 388-394 (2010).

18. R. Vogel, M. Persson, C. Feng, S. J. Parkin, T. A. Nieminen, B. Wood, N. R. Heckenberg, and H. Rubinsztein-Dunlop, "Synthesis and surface modification of birefringent vaterite microspheres," Langmuir 25, 1167211679 (2009).

19. K. Dholakia and P. Zemanek, "Colloquium: Gripped by light: Optical binding," Rev. Mod. Phys. 82, 1767 (2010).

20. S. A. Tatarkova, A. E. Carruthers, and K. Dholakia, "One-dimensional optically bound arrays of microscopic particles," Phys. Rev. Lett. 89, 283901 (2002).

21. M. M. Burns, J. M. Fournier, and J. A. Golovchenko, "Optical matter crystallization and binding in intense optical-fields," Science 249, 749754 (1990).

22. M. Guillon, O. Moine, and B. Stout, "Longitudinal optical binding of high optical contrast microdroplets in air," Phys. Rev. Lett. 96, 143902 (2006).

23. M. Guillon and B. Stout, "Optical trapping and binding in air: Imaging and spectroscopic analysis," Phys. Rev. A 77, 023806 (2008). 